\title{
The Pemba fruit bat-on the edge of extinction?
}

\author{
Ole Seehausen
}

The population of endemic fruit bats on Pemba Island, which lies off the coast of Tanzania, appears to have undergone a drastic decline. The author made a short survey in 1989 in some of the areas where the fruit bat was reported to have been numerous, but found that few now exist in these places. The change from traditional hunting methods to the use of shotguns as well as destruction of the island's rain forest are believed to be the principal causes. The author makes a plea for a ban on hunting, a public education campaign, protection of the surviving remnants of forest and a captive-breeding effort as a safeguard against extinction.

The islands in the western Indian Ocean are an important centre of endemism for the large fruit bats of the genus Pteropus (Kingdon, 1974). Most islands or groups of islands between the Seychelles in the north and Madagascar in the south have endemic species or subspecies of these fruit bats (Hill, 1971; Carroll, 1985). Of these, one, Pteropus subniger, from Mauritius and Reunion is extinct. Two others known to be endangered- $P$. rodricensis from Rodrigues island and $P$. livingstonii from Anjouan and formerly Moheli, Comoro islands-have received some attention from the conservation community (Wirth and Riffel, 1989). However little is known of the status of most of the other endemic forms.

In late October/early November 1989, I spent a week on Pemba island, off the coast of Tanzania, north of Zanzibar, to obtain information on the population status of the endemic fruit bat Pteropus voeltzkowi. Pakenham (1984), writing of the period 1938-45, reports of hundreds of these bats roosting on islands off the south coast and off north-west Pemba, crossing at dusk to spread over southern and northern Pemba. Despite the short duration of my project, which precludes the possibility of making a definitive assessment of the present status of these animals, it is nevertheless clear that Pteropus voeltzkowi has undergone a dramatic decline in recent years. Kingdon (1974) referred to a roosting site, Matanga-Mgogoni on Pemba (relating to the area between two villages, Mgogoni and Matangatuani, in northern Pemba [J. Kingdon, pers. comm.]) close to the last vestiges of forest on the island. However, despite extensive searches made with the help of local villagers, no fruit bats were seen in the vicinity of Mgogoni and the nearby Ngezi forest, although the same villagers reported that they still occasionally see up to 50 fruit bats flying eastwards in the evenings from remaining forest patches on some of the steep hillsides to the west. Several of these forested slopes were also searched, but no fruit bats were located.

Other areas investigated for the continuing presence of this species included Mzambaraoui and Kisiwani (Figure 1). In Mzambaraoui the survey was assisted by teachers of the Piki primary school, who also interviewed people in surrounding villages. However, no reports of the local occurrence of fruit bats were obtained by this means, and it seems that they no longer occur in this region. In Kisiwani-Kijumbani, local informants reported that $P$. voeltzkowi was formerly common but is rarely seen nowadays. A large aggregation of animals was last seen 10 years ago in an Eugenia malaccensis tree approximately $3 \mathrm{~km}$ east of the village. This tree was in fruit at the time of my visit, but no fruit bats were located. However after extensive searches, three bats were seen on 27 September in a tree close to some mangroves. Sight checks of other 
fruiting trees during the night failed to reveal any other bats, but solitary flying individuals were seen in this region on three occasions. The only other location where a bat was sighted was south of Ziwani, where another single animal was seen in flight (Figure 1). A few fruit bats are also said to still exist around Chake/Machumani, where they were reported to have been quite common in the past.

$P$. voeltzkowi has been traditionally hunted for food on Pemba. In the past the bats were hunted with nooses attached to long sticks with which they were plucked from roosting

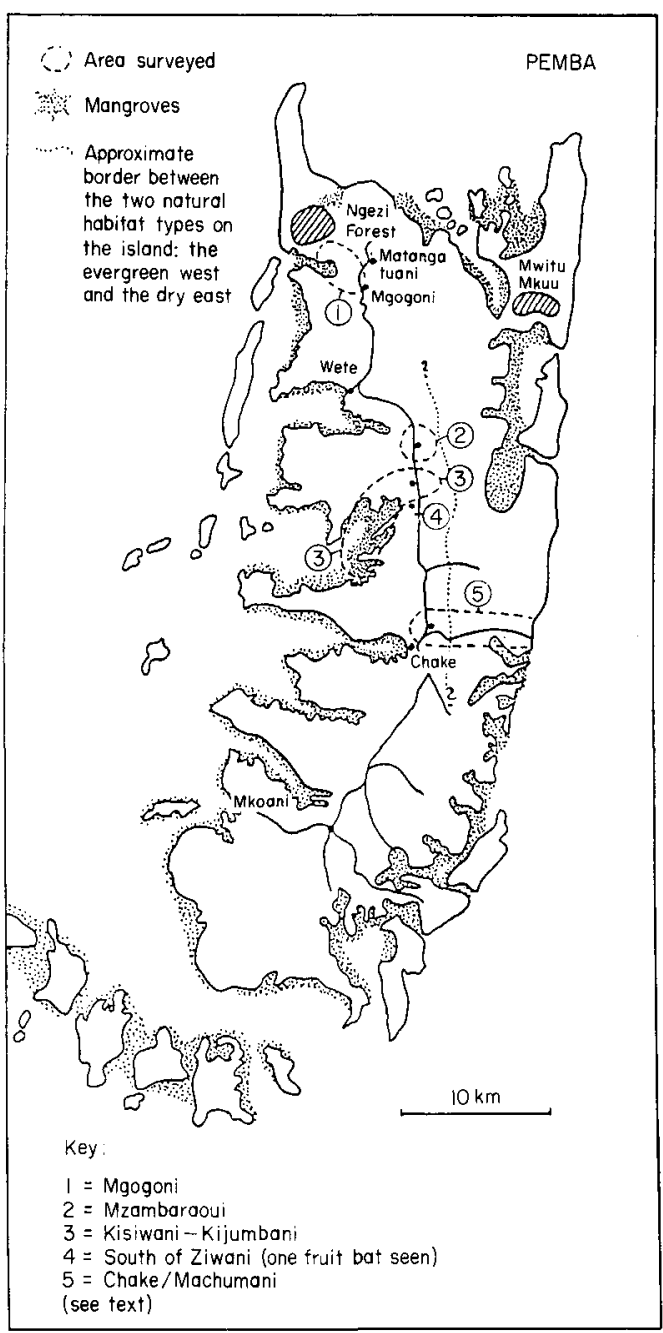

Figure 1. Pemba Island showing area surveyed and habitat types. trees like ripe fruits. Occasionally, roosting individuals were also caught by throwing cudgels. These methods were unlikely to have caused a dramatic decline in the population, as any such disturbance would have induced the colony to fly off, so only a few animals could have been collected at any one time. However, the introduction of hunting with shot guns meant that as many as 20 bats could be killed with a single shot of lead pellets. $K$. Salim (principal of Piki primary school) attributes the virtual disappearance of the species in the Mzambaraoui and Kisiwani regions to hunting with shot guns.

Hunting may, therefore, be one of the principal causal factors in the evident recent decline of $P$. voeltzkowi, but habitat destruction must also have an important role. The western half of Pemba was formerly covered with tropical rain forest, whereas the eastern half was dominated by baobab Adansonia digitata and Borassus palm savannahs and coastal bush. As far as could be ascertained from local people the fruit bat was endemic to the rain forest, and it is reported to have originally occurred throughout the western half of the island, but not in the dry east. Much of the original rain forest was cleared for agriculture, particularly clove plantations, in the eighteenth century, and Pemba is now rather densely populated (approximately 200 people/sq $\mathrm{km}$ ). Nevertheless, as long as some patches of natural forest remained on hill slopes and traditional hunting methods were used, the fruit bat seemed to have survived in reasonable numbers. However, the last fragments of natural forest are now in danger of disappearing and years of hunting with lead pellets (a practice that continues) has apparently reduced the population to a critically low level.

Pemba Island is biogeographically very interesting with faunistic and floristic elements linking East Africa with the Mascarenes and other islands in the Indian Ocean (Moreau and Pakenham, 1941). Numerous subspecies and several species are endemic to the island. Large fruit bats are now being recognized as important pollinators and seed dispersers in tropical ecosystems. P. voeltzkowi could and should, therefore, be a flagship species for 
conservation initiatives on Pemba, which are required urgently. The 'island world' between the Pacific and Indonesia (including the Philippines with about 40 out of 55 species) appears to be the centre of Pteropus evolution (Kingdon, 1974), and P. voeltzkowi is also of some interest in being geographically most remote from that presumed centre of radiation.

Efforts to save the fruit bat and what else remains of the original biological wealth of Pemba should include public education on the island, a ban on hunting, and official protection of the few surviving rain forest fragments. In the interim, captive breeding should also be considered a high priority and efforts made to secure a founder population in the near future.

\section{Acknowledgments}

I thank Abduu Muhammad Salim, Khamis and Mama Dhow Salim and their family, as well as Ashirak Salim, who helped during my stay in Pemba in many ways and walked uncounted kilometres with me in search of the fruit bats. William
Oliver and Roland Wirth read and commented on the draft manuscript.

\section{References}

Carroll, J.B. 1985. The flying foxes (Pteropus spp.) of the western Indian Ocean islands and a footnote on Rousettus obliviosus. Bat News, 4, 4-5.

Hill, J.E. 1971. The Bats of Aldabra Atoll, Western Indian Ocean. Phil. Trans. Roy. Soc. Lond. B.260, 573-576.

Kingdon, J. 1974. East African Mammals: An Atlas of Evolution in Africa. IIA (Insectivores and Bats). Academic Press, London and New York.

Moreau, R.E. and Pakenham, R.H.W. 1941. The land vertebrates of Pemba, Zanzibar and Mafia: a zoogeographical study, Proc. Zool. Soc. London, (A) 110, 97-128.

Pakenham, R.H.W. 1984. The Mammals of Zanzibar and Pemba Islands. Privately published by R. H.W. Pakenham.

Wirth, R. and Riffel, M. 1989. Bedrohte Flughunde, Newsletter of the Zoological Society for the Conservation of Species and Populations, March 1989.

Ole Seehausen, Lavesstr. 18, 3000 Hannover 1, Germany. 